\title{
Monographien der Abteilung Prävention
}

Eine präventive zeitlich wenig anspruchsvolle Intervention in der Sprechstunde zeitigt oft grosse Wirkung! In Broschüren und Basisdokumentationen der Abteilung Prävention finden Sie dazu viel Wissenswertes zu Themen wie "Frei von Tabak», "Krise und Suizid», "HIV», "Kreuzschmerzen».

Une intervention préventive peu chronophage peut s'avérer efficace! Les brochures et manuels de la division prévention vous donnent toutes les informations importantes pour aborder les thèmes "Vivre sans tabac», "Crise et suicide», "VIH», "Lombalgies».

St

Frei von Tabak / Vivre sans tabac

neue Basisdokumentation / nouvelle Documentation de base

Fr. 20.45 (inkl. MwSt)

$\square \mathrm{D} \quad \square \mathrm{F} \quad$ Ex.

Ärzte und Tabak, die grosse medizinische Herausforderung

kostenlos / gratuit

$\square \mathrm{F} \quad \square \mathrm{I} \quad \mathrm{Ex}$.

Krise und Suizid / Crise et suicide

neue Basisdokumentation / nouvelle Documentation de base

Fr. 15.35 (inkl. MwSt)

$\square \mathrm{D} \quad \square \mathrm{F} \quad$ Ex.

Patientenbroschüren / brochures pour patients

kostenlos / gratuit

$\square \mathrm{D} \quad \square \mathrm{F} \quad \square \mathrm{I} \quad-\mathrm{Ex}$

Reminder

$\square \mathrm{D} \quad \square \mathrm{F} \quad \square \mathrm{I} \quad \mathrm{Ex}$.

kostenlos / gratuit

HIV/VIH

Basisdokumentation / Documentation de base

Fr. 20.45 (inkl. MwSt)

$\square \mathrm{D} \quad \square \mathrm{F} \quad \square \mathrm{I} \quad$ Ex.

BACK in time: Kreuzschmerzen / Lombalgies

Algorithmen / Algorithmes

Fr. 15.35 (inkl. MwSt)

$\square \mathrm{D} \quad \square \mathrm{F} \quad \square \mathrm{I} \quad$ Ex.

Basisdokumentation / Documentation de base

Fr. 20.45 (inkl. MwSt)

$\square \mathrm{D} \quad \square \mathrm{F} \quad$ Ex.

Kombination Algorithmen + Basisdokumentation /

Combinaison Algorithmes + Documentation de base

Fr. 30.70 (inkl. MwSt)

$\square \mathrm{D} \quad \square \mathrm{F} \quad$ Ex.

Name / Nom:

Vorname / Prénom:

Adresse:

zurücksenden an/envoyer à:

Generalsekretariat FMH, Abteilung Prävention, Frau B. Weil, Elfenstrasse 18, 3000 Bern 16,

Fax 03135911 12, E-mail: bweil@hin.ch 